\title{
Erratum: Subdiffusive continuous-time random walks with stochastic resetting [Phys. Rev. E 99, 052116 (2019)]
}

\author{
Łukasz Kuśmierz (1) and Ewa Gudowska-Nowak
}

(Received 6 February 2020; accepted 7 February 2020; published 26 February 2020)

DOI: 10.1103/PhysRevE.101.029904

In this paper, we have analyzed a continuous-time random walk (CTRW) model of subdiffusion with a "soft" stochastic resetting where reset events bring the particle back to the initial position but do not affect the waiting times. The analysis of the mean square displacement (MSD) in this model exhibits a nonmonotonic behavior which has been commented (cf. p. 7): ..."nonmonotonic behavior of the process relies on the trapping with power-law waiting times" and "we hypothesize that this [nonmonotonic $\left\langle X^{2}(t)\right\rangle$ behavior] is related to the weak ergodicity breaking"...

In a discussion following the publication, it was brought to our attention by V. P. Shkilev [1] that the hypothesis on the direct relation between ergodicity breaking and the nonmonotonicity of the MSD can be easily invalidated. Indeed, it is enough to consider waiting times generated from a biexponential distribution [2] and solve the renewal equation for the corresponding propagator of the CTRW under Poissonian resetting. Such a process is ergodic, but there exists a parameter regime in which a nonmonotonic behavior of the $\operatorname{MSD}\left\langle X^{2}(t)\right\rangle$ can be detected.

We would like to acknowledge V. P. Shkilev (Chuiko Institute of Surface Chemistry, National Academy of Sciences of Ukraine) for stimulating exchanges on this case and bringing our attention to various sources of nonmonotonicity of the MSD in processes with resetting.

[1] V. P. Shkilev (private communication).

[2] V. P. Shkilev, Phys. Rev. E 96, 012126 (2017). 